\title{
Heart Failure in Adult Patients with Congenital Heart Disease
}

\author{
Valérie M. Smit-Fun, MD*, Wolfgang F. Buhre, MD, PhD
}

\section{KEYWORDS}

- Adult congenital heart disease $\bullet$ Grown-up congenital heart disease $\bullet$ Heart failure

- Fontan circulation • Single ventricle

\section{KEY POINTS}

- Almost $90 \%$ of children born with a congenital heart disease survive into adulthood and achieve an average age of almost 60 years.

- With increasing age the care for adult patients with congenital heart disease becomes more challenging as residual cardiac defects, superimposed by acquired disease, result in complex morbidity.

- Heart failure has a high prevalence in adults with congenital heart disease and is the main cause of mortality; knowledge of pathophysiology and prevention of complications is essential.

- Care for adults with congenital heart disease is not restricted to highly specialized centers, although it is recommended to manage patients with complex morbidity in specialized centers with multidisciplinary expert teams.

\section{INTRODUCTION}

Owing to advances in cardiac surgery, intensive care, and diagnostic capabilities, $88 \%$ of children born with a congenital heart disease (CHD) survive into adulthood. ${ }^{1-3}$ Besides arrhythmias, (end-stage) heart failure is the primary source of morbidity and mortality. In accordance with the increasing number of adult patients with $\mathrm{CHD}$ $(\mathrm{ACHD})^{\mathrm{a}}$, the need for noncardiac surgical and interventional procedures is increasing, both in the elective and acute settings. Therefore, there is a practical need for anesthesiologists to be familiar with the pathophysiologic considerations. In this review,

\footnotetext{
Disclosure Statement: None.

Department of Anaesthesiology and Pain Medicine, Maastricht University Medical Center, PO Box 5800, Maastricht 6202 AZ, The Netherlands

* Corresponding author.

E-mail address: v.smit.fun@mumc.nl
}

a There are some inconsistencies regarding the nomenclature. In some countries the term "grown-up congenital heart disease" is used (GUCH). The reference to this patient population as "adults with congenital heart disease" (ACHD) is more common. 
we describe the actual knowledge about heart failure in this heterogeneous patient population. Special attention is given to patients with a Fontan circulation, for example, the cohort with a single ventricle circulation and passive pulmonary circulation. Furthermore, general considerations regarding the increasingly present obstetric and surgical patient populations with ACHD are discussed. There are only a couple of controlled clinical trials available, and, thus, our knowledge is based primarily on single-center experiences, case series, and small-scale observational clinical studies.

\section{CONGENITAL HEART DEFECTS}

Structural heart disease is the most common congenital disorder in newborns. The pathophysiology of $\mathrm{CHD}$ is heterogeneous and consists of a great range of cardiac anomalies. The 8 most common CHD subtypes and their birth prevalence ${ }^{4}$ are shown in Fig. 1.

The complexity of the congenital defect, the possibility of surgical correction or definitive palliation largely determine morbidity and mortality in patients with CHD.

Usually, the Bethesda Classification of Congenital Heart Disease is used to describe the severity of CHD in terms of complexity. The definition was developed at a consensus meeting in 2000 , and is still applicable and widely used. In 2018, Stout and colleagues ${ }^{5}$ published the actual guideline for the management of ACHD including

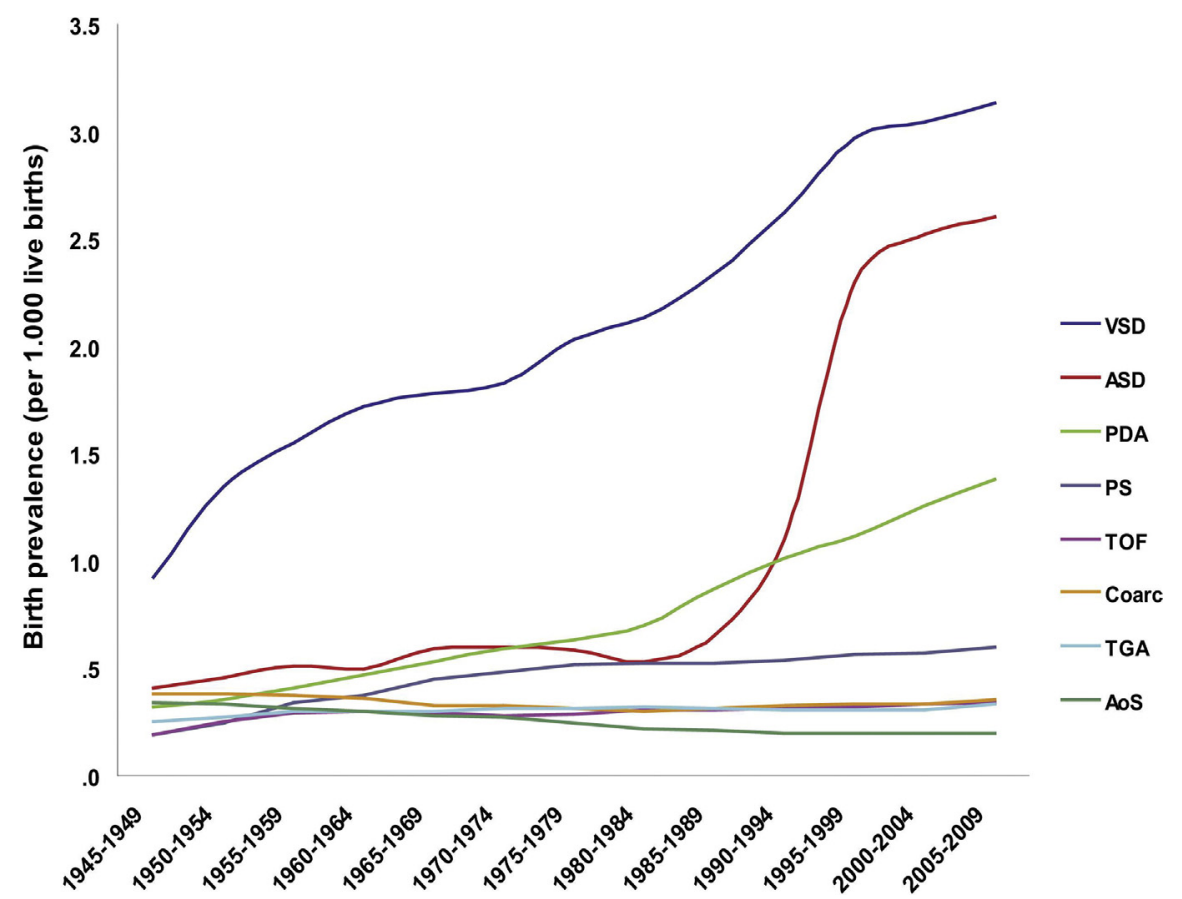

Fig. 1. Birth prevalence of CHD subtypes over time. Time course of birth prevalence of the 8 most common CHD subtypes from 1945 until 2010. AoS, aortic stenosis; ASD, atrial septal defect; Coarc, coarctation; PDA, patent ductus arteriosus; PS, pulmonary stenosis; TGA, transposition of the great arteries; TOF, tetralogy of Fallot; VSD, ventricular septal defect. (From van der Linde D, Konings EE, Slager MA, et al. Birth Prevalence of Congenital Heart Disease Worldwide: a systematic review and meta-analysis. J Am Coll Cardiol 2011;58(21):2245; with permission.) 
the classification of disease and the relation to the New York Heart Association (NYHA) functional classification of heart failure (Box 1). According to complexity, 3 groups of patients with CHD are identified: those with simple defects, moderately complex defects, and complex defects. Approximately $3 \%$ of live births with CHD are complex defects and $15 \%$ are moderately complex defects.

\section{EPIDEMIOLOGY}

The incidence of CHD is 3 to 20 per 1000 live births, resulting in a prevalence of 6.9 in North America, 8.2 in Europe, and 9.3 per 1000 live births in Asia. ${ }^{4,6}$ In infants and children, complexity and surgical results have been shown to be the primary determinants of mortality. ${ }^{1-3}$ Owing to advances in cardiac surgery, intensive care, and diagnostic capabilities, $88 \%$ of patients survive into adulthood. ${ }^{1-3}$ The reported average age for patients with ACHD in 2007 was 57 years. ${ }^{2,7}$ Data from Afilalo and colleagues ${ }^{8}$ showed that the aged population older than 65 years is still substantial with a similar prevalence as in the adult population of 3 per thousand elderly.

Despite surgical correction, patients with CHD carry a life-long risk for cardiovascular complications arising from residual defects and the clinical course of the disease. Moreover, for some patients no corrective approach is possible resulting in a stage of definitive palliation.

Agarwal and colleagues ${ }^{9}$ studied trends in resource use from 2003 to 2012 and noted a significant increase in the number of ACHD related emergent and nonemergent hospital admissions, with almost 30\% presenting to small- and medium-sized hospitals, whereby $25 \%$ represented complex ACHD. In 2012, compared with 2003, there was a considerable increase in the prevalence of traditional cardiovascular risk factors (hypertension, diabetes, smoking, obesity, chronic kidney disease, and peripheral arterial disease), a significant increase in length of stay, and a considerable increase in cost of admission for both simple and complex patients with ACHD. In this study cohort, in-hospital mortality remained relatively constant.

Heart failure is responsible for approximately $13 \%$ of hospitalizations, with other main reasons being valve disease (15\%) and cerebrovascular accidents (26\%). ${ }^{7,9}$ Hospital admission caused by heart failure is associated with a 5-fold increase in mortality ${ }^{10}$ and is the main cause of death in ACHD (20\%). ${ }^{11}$ Patients admitted with heart failure are significantly older and generally have more complex ACHD. ${ }^{7}$

\section{HEART FAILURE IN ADULT CONGENITAL HEART DISEASE Causes of Heart Failure in Patients with Congenital Heart Disease}

In patients with acquired cardiac disease, heart failure mainly results from left ventricle (LV) systolic or diastolic dysfunction caused by ischemia, valve disease, or even cardiomyopathy. In ACHD heart failure the underlying mechanism is more heterogenous and includes chronic pressure and/or volume (over)loading, inadequate myocardial preservation during prior surgeries, myocardial fibrosis, surgical injury to a coronary artery, pulmonary hypertension, and neurohormonal activation. Furthermore, a subset of patients can develop symptoms of heart failure in the time course after initial successful surgical correction of a congenital anomaly. In a variety of settings the right ventricle $(R V)$ acts as the systemic ventricle which ultimately can lead to an increased incidence of RV systemic failure.

Thus, in patients with ACHD, RV failure is a common phenomenon with an incidence of up to $70 \%$ in selected patient populations. Moreover, some patients suffer from residual shunt, which can lead to increased pulmonary blood flow in case of a left-toright shunt, thus, resulting in pulmonary hypertension, ultimately leading to RV 


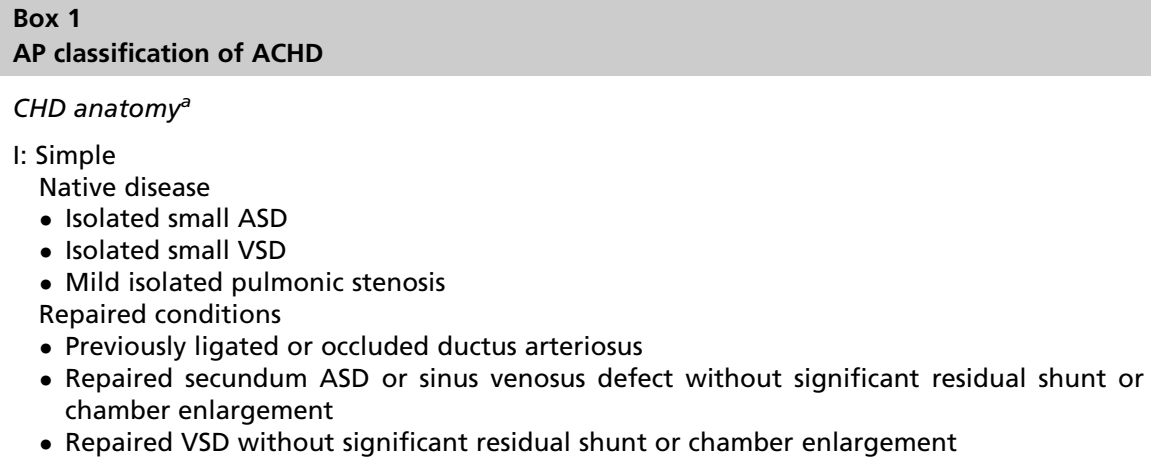

II: Moderate complexity repaired

Repaired or unrepaired conditions

- Aorto-left ventricular fistula

- Anomalous pulmonary venous connection, partial or total

- Atrioventricular septal defects (partial or complete, including primum ASD)

- Coarctation of the aorta

- Ebstein anomaly (disease spectrum includes mild, moderate, and severe variations)

- Infundibular right ventricular outflow obstruction

- Ostium primum ASD

- Moderate and large persistently patent ductus arteriosus

- Pulmonary valve regurgitation (moderate or greater)

- Pulmonary valve stenosis (moderate or greater)

- Sinus of Valsalva fistula/aneurysm

- Sinus venosus defect

- Subvalvar or supravalvar aortic stenosis

- Repaired tetralogy of Fallot

- Ventricular septal defect with associated abnormality and/or moderate or greater shunt

III: Great complexity (or complex)

- Cyanotic congenital heart defect (unrepaired or palliated, all forms)

- Double-outlet ventricle

- Fontan procedure

- Mitral atresia

- Single ventricle (including double inlet left ventricle, tricuspid atresia, hypoplastic left heart, any other anatomic abnormality with a functionally single ventricle)

- Pulmonary atresia (all forms)

- TGA (classic or d-TGA; cc-TGA or I-TGA)

- Truncus arteriosus

- Other abnormalities of atrioventricular and ventriculoarterial connection (ie, crisscross heart, isomerism, heterotaxy syndromes, ventricular inversion)

\section{Physiologic stage $A$}

NYHA FC I symptoms

- No hemodynamic or anatomic sequelae

- No arrhythmias

- Normal exercise capacity

- Normal renal/hepatic/pulmonary function

Physiologic stage $B$

NYHA FC II symptoms

- Mild hemodynamic sequelae (mild aortic enlargement, mild ventricular enlargement, mild ventricular dysfunction)

- Mild valvular disease

- Trivial or small shunt (not hemodynamically significant)

- Arrhythmia not requiring treatment

- Abnormal objective cardiac limitation to exercise 
Physiologic stage $C$

NYHA FC III symptoms

- Significant (moderate or greater) valvular disease; moderate or greater ventricular dysfunction (systemic, pulmonic, or both)

- Moderate aortic enlargement

- Venous or arterial stenosis

- Mild or moderate hypoxemia/cyanosis

- Hemodynamically significant shunt

- Arrhythmias controlled with treatment

- Pulmonary hypertension (less than severe)

- End-organ dysfunction responsive to therapy

Physiologic stage $D$

NYHA FC IV symptoms

- Severe aortic enlargement

- Arrhythmias refractory to treatment

- Severe hypoxemia (almost always associated with cyanosis)

- Severe pulmonary hypertension

- Eisenmenger syndrome

- Refractory end-organ dysfunction

CHD Anatomy + Physiologic stage $=$ ACHD AP classification.

Abbreviations: AP, anatomic and physiologic; ASD, atrial septal defect; cc-TGA, congenitally corrected transposition of the great arteries; d-TGA, dextro-transposition of the great arteries; FC, functional class; I-TGA, levo-transposition of the great arteries; TGA, transposition of the great arteries; VSD, ventricular septal defect.

${ }^{a}$ This list is not meant to be comprehensive; other conditions may be important in individual patients.

Adapted from Stout KK, Daniels CJ, Aboulhosn JA, et al. 2018 AHA/ACC Guideline for the Management of Adults With Congenital Heart Disease: Executive Summary: A Report of the American College of Cardiology/American Heart Association Task Force on Clinical Practice Guidelines. J Am Coll Cardiol 2019;73(12):1504; with permission.

dysfunction secondary to pressure overload. ${ }^{12-15}$ The prevalence of ACHD-heart failure is highest in patients with complex anatomy, including single ventricle physiology, transposition of the great arteries (TGA), tetralogy of Fallot (TOF), and pulmonary hypertension. ${ }^{13}$

Patients with ACHD are generally younger when developing heart failure and often the decrease in cardiac function is accompanied by tachyarrhythmias of a different origin. Basically, advanced rhythm therapy can contribute to treating heart failure appropriately.

\section{Diastolic Heart Failure}

During the past years, there has been an increasing focus on LV heart failure with preserved systolic LV (diastolic) function, or diastolic heart failure. In the population of patients with ACHD, information on diastolic LV failure is relatively sparse.

\section{Diagnosis of Heart Failure in Adult Patients with Congenital Heart Disease}

\section{Signs and symptoms}

Heart failure is a clinical diagnosis. It is defined as a syndrome of signs and symptoms of cardiac congestion caused by a structural and/or functional cardiac abnormality, resulting in reduced cardiac output and/or elevated intracardiac pressures at rest or during stress. ${ }^{5,16,17}$ 
Patients with ACHD themselves tend to underreport their cardiac symptoms because they are used to living with cardiac disease. At the time of symptom recognition, the extent of ventricular dysfunction and valve disease may be severe and irreversible. Considering the high mortality of ACHD heart failure, it is recommended to pay specific attention to signs of developing heart failure and perform diagnostic tests during follow-up to initiate therapy in an early stage of ACHD-heart failure.

Diagnostic and therapeutic recommendations in guidelines are based on the majority of heart failure, which is usually caused by LV systolic or diastolic dysfunction in patients without CHD. However, heart failure in CHD is more often a consequence of RV disease, valve dysfunction, shunting or pulmonary hypertension. The evaluation and treatment of heart failure in CHD should therefore be based on the specific structural and functional circulatory lesion. Recently, a task force of the American Heart Association and the American College of Cardiologists published the first guideline for the management of ACHD. ${ }^{5}$

\section{Diagnosis}

In general, patients with ACHD should be treated by specialist cardiologists. Within the last years, cardiac centers have established specialized ambulatory care units dedicated to these patients. Within such centers, a wide range of technical and clinical diagnostics is available. The basic assumptions for diagnosing heart failure is the combination of clinical examination, functional capacity testing, and technical investigations combined with biomarker monitoring.

Functional capacity Cardiopulmonary exercise testing is often considered the gold standard for quantitative assessment of functional capacity. It correlates well with NYHA functional classification, functional capacity, and anaerobic threshold during cardiopulmonary exercise testing. A subnormal peak $\mathrm{V}_{2}$ during cardiopulmonary exercise testing has been demonstrated in asymptomatic ACHD, highlighting the need for the evaluation of heart failure even in the absence of symptoms. ${ }^{18}$

The NYHA Functional Classification (see Box 1) is commonly used to describe the functional capacity in heart failure ${ }^{18}$ and correlates well with the severity of heart failure and strategies for treatment, and ultimately predicts mortality. ${ }^{5,18}$ It was however questionable whether this was also applicable for patients with ACHD. Recently, Bredy and colleagues ${ }^{18}$ demonstrated that the NYHA functional class correlated strongly with predictive capacity of exercise testing and with the Bethesda classification in ACHD.

Cardiac imaging Echocardiography is a noninvasive technique for monitoring disease progression and provides assessment of intracardiac anatomy and overall cardiac function at rest and during exercise. Specific guidelines for echocardiographic evaluation of RV pressures and volumes in ACHD are available. ${ }^{19}$

Cardiac magnetic resonance imaging has the ability to provide quantitative assessment of blood flow, valvular regurgitation, chamber volumes and ventricular function. Also, anatomic and functional cardiac assessment during pharmacologic stress testing is possible with a stress MRI. In the presence of contraindications to cardiac magnetic resonance imaging, a cardiac computed tomography scan is an alternative with the ability to quantify ventricular volumes and assess ventricular function.

Cardiac biomarkers At baseline, elevated levels of atrial natriuretic peptide, brain-type natriuretic peptide, $N$-terminal pro-brain-type natriuretic peptide (NT-proBNP), endothelin-1, norepinephrine, renin, and aldosterone have been found in ACHD. Several studies have shown a relationship between the degree of cardiac biomarker 
elevations, NYHA functional class, and systemic ventricular function. ${ }^{3,12}$ The degree of elevation varies between ACHD lesions.

\section{Management of Heart Failure in Adult Patients with Congenital Heart Disease}

\section{General considerations}

Guidelines published by the American College of Cardiology/American Heart Association/Heart Failure Society of America and the European Society of Cardiology on the management of heart failure in acquired heart disease are readily available. These recommendations emphasize the use of neurohormonal blockade with angiotensinconverting enzyme inhibitors, angiotensin receptor blockers (ARB), and betablockers, which have proven morbidity and mortality benefits in heart failure. ${ }^{17}$ Management of this complex ACHD population has largely been extrapolated from literature that is based on adults with acquired heart disease. Very recently, the American College of Cardiology/American Heart Association guidelines for the management of $\mathrm{ACHD}$ in general were published, but recommendations for the specific management of heart failure in ACHD are scarce. For further reading on heart failure in ACHD, 2 major publications from Stout and colleagues ${ }^{14}$ and Budts and colleagues ${ }^{20}$ are recommended.

\section{Subtypes of heart failure in adult patients with congenital heart disease}

Systolic dysfunction of the systemic left ventricle Systolic dysfunction in the systemic LV results from coronary artery disease, systolic dysfunction of the LV as a consequence of volume loading (aortic regurgitation, mitral regurgitation, and residual ventricular septal defect) or pressure loading (aortic stenosis, and coarctation of the aorta) (Table 1). ${ }^{3,5,12,14,15,17}$ Diagnosis and treatment is in line with the general American College of Cardiology/American Heart Association heart failure guidelines for patients without ACHD. ${ }^{16}$

Systolic dysfunction of the systemic right ventricle In some types of ACHD, the anatomic right ventricle acts as the ventricle serving the systemic circulation. Classical examples are congenitally corrected TGA, as well as surgically corrected TGA (d-TGA) after the Mustard or Senning atrial switch operations (Table 2). RV failure has been shown to develop in $67 \%$ of patients with congenitally corrected TGA by the age of 45 years, and in $12 \%$ of patients with d-TGA 12 years after the atrial switch procedure. Overall, heart failure is the leading cause of death in up to $66 \%$ of patients with a systemic RV. $3,12,14$

The effectiveness of guideline-directed medical therapy in decreasing morbidity or mortality in systemic RV failure is unclear. Some studies with angiotensin-converting enzyme inhibitor treatment have noted a trend toward improved peak $\mathrm{V}_{2}$ and a decrease in levels of NT-proBNP, but evidence for significant improvements in exercise peak $\mathrm{VO}_{2}$, tricuspid regurgitation, $\mathrm{RV}$ size, or $\mathrm{RV}$ systolic function is lacking. Studies involving ARBs have demonstrated a lack of benefit. Increased catecholamine levels in patients with systemic RV and increases in concentrations of epinephrine and norepinephrine correlate with increased cardiothoracic ratio and RV diastolic dimensions. In small studies the use of beta-blockers have demonstrated improvements in quality of life and NYHA functional class. Treatment with higher doses of beta-blockers in patients with d-TGA and cardiac pacemakers failed to demonstrate improvement in RV size and systolic function, but showed significant improvements in NYHA functional class. ${ }^{3,5,12-15,17}$

Tachycardia-mediated cardiomyopathy is an important cause of systemic RV failure. In patients after an atrial switch procedure for d-TGA, the incidence of arrhythmia increases over time, from nearly $78 \%$ arrhythmia-free survival at 10 years to only $36 \%$ 


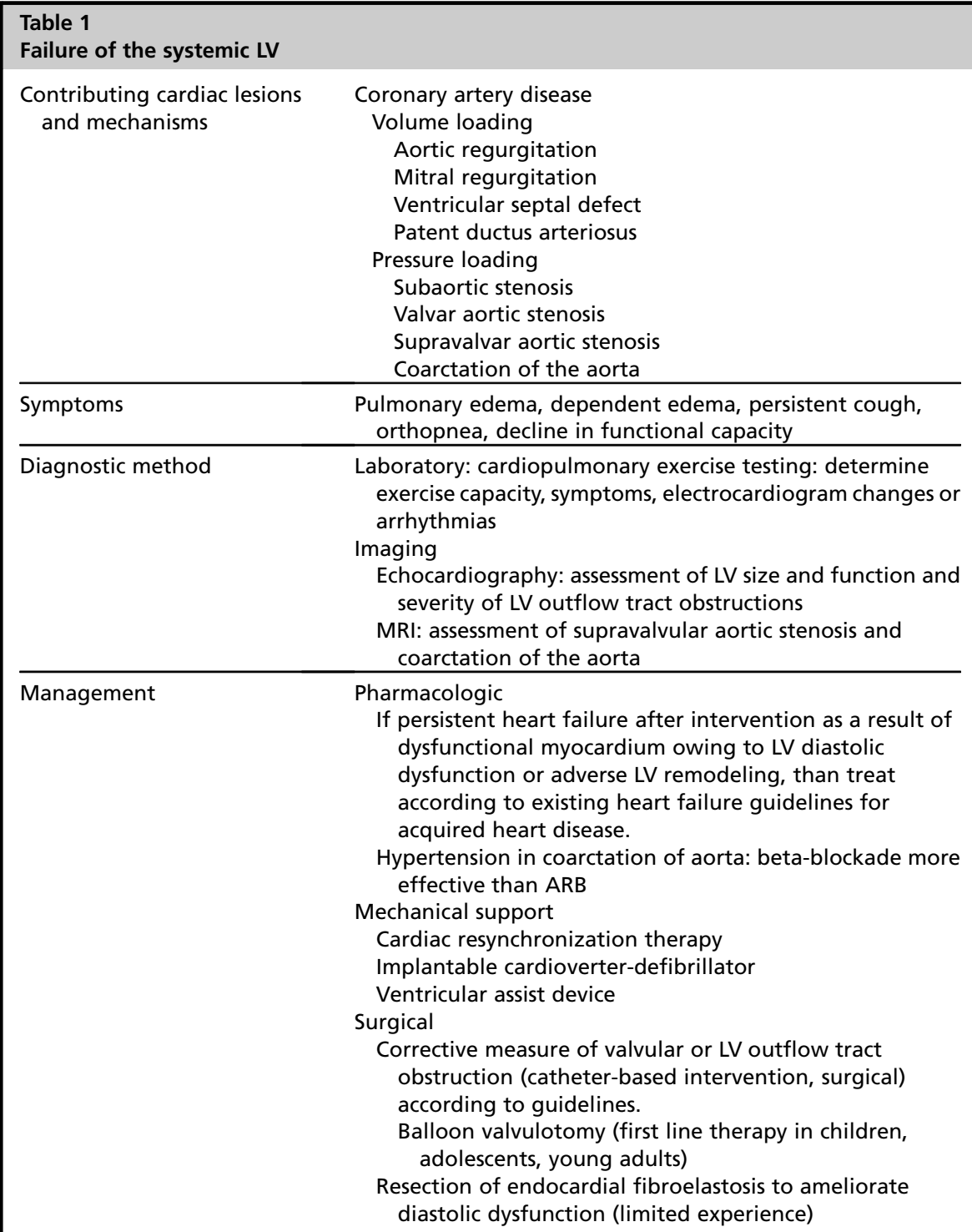

at 25 years after the procedure. In these patients, intense monitoring of heart rhythm is justified. It remains unknown if modern electrophysiology approaches may result in improved outcome in these patients. , $^{3,5}, 12-14$

Right ventricular dysfunction RV dysfunction is most common in patients with Tetralogy of Fallot, but can develop in a variety of other cardiac lesions caused by rightsided volume and/or pressure overload (Table 3). Pulmonary stenosis and pulmonary hypertension are the most common pressure-loading lesions that have the potential to result in subpulmonic RV failure. A number of $\mathrm{CHD}$ types are characterized by outflow obstruction of the RVOT with increasing risk of RV failure. . $^{3,12,14}$ 


\begin{tabular}{|c|c|}
\hline $\begin{array}{l}\text { Contributing cardiac } \\
\quad \text { lesions and mechanisms }\end{array}$ & $\begin{array}{l}\text { Congenitally corrected TGA } \\
\text { D-TGA }\end{array}$ \\
\hline Symptoms & $\begin{array}{l}\text { Pulmonary edema, dependent edema, persistent cough, } \\
\text { orthopnea, decline in functional status, chronotropic } \\
\text { incompetence, atrial and ventricular tachyarrhythmia }\end{array}$ \\
\hline Diagnostic method & $\begin{array}{l}\text { Annual clinical evaluation, more frequent as necessary } \\
\text { Laboratory, brain-type natriuretic peptide: level correlates with } \\
\text { deterioration in clinical status, declining RV ejection fraction, } \\
\text { decreasing exercise capacity, worsening TR. Cut off value not } \\
\text { defined. } \\
\text { Imaging, periodic (RV function, TR): } \\
\text { Echocardiography } \\
\text { MRI/cardiac MRI (RV size and function, TR severity, myocardial } \\
\text { fibrosis) } \\
\text { Stress cardiac MRI (inability to increase RV ejection fraction } \\
\text { predictive for cardiac events) }\end{array}$ \\
\hline Management & $\begin{array}{l}\text { Pharmacologic } \\
\text { Conclusive data are lacking. } \\
\text { Beware of preexisting sinus node dysfunction, heart block, } \\
\text { baffle stenosis, nondistensible atria, restrictive RV } \\
\text { physiology } \\
\text { Angiotensin-converting enzyme inhibitors/ARB: no } \\
\text { improvement in ejection fraction } \mathrm{Vo}_{2}, \mathrm{Cl} \\
\text { Beta-blockade: shown to improve RV function and } \\
\text { symptoms, less systemic TR, improve functional status, } \\
\text { positive effect RV remodeling, protection against } \\
\text { arrhythmia } \\
\text { Mechanical support } \\
\text { Implantable cardioverter-defibrillator: consider for primary } \\
\text { prevention, although only after or in conjunction with beta- } \\
\text { blockers to mitigate arrhythmias } \\
\text { CRT: management of brady- and tachyarrhythmia. Benefit } \\
\text { unknown; modest improvement in RVEF and NYHA } \\
\text { suggested } \\
\text { Surgical } \\
\text { Treat residual such as outflow obstruction or valvular } \\
\text { regurgitation before significant valvular dysfunction. } \\
\text { Ventricular assist device and CHD transplantation: systolic RV } \\
\text { failure who fail medical therapy and without residual } \\
\text { lesions. }\end{array}$ \\
\hline
\end{tabular}

Abbreviations: CRT, cardiac resynchronization therapy; TR, tricuspid regurgitation.

Echocardiography is the most common used diagnostic measure in these patients. In addition, cardiac MRI is sometimes indicated. Assessment of peak exercise capacity helps to document the course of the disease. . $^{3,5,14,15,17}$

Surgical or percutaneous intervention aimed at correcting these volume/pressure loading conditions offer the best chance of improving ventricular function, but the timing of such interventions is a matter of debate. Pharmacologic therapy with pulmonary vasodilators (phosphodiesterase- 5 inhibitors, endothelin-receptor antagonists, or prostacyclin analogs) form the basis of pulmonary hypertension management, and diuretics can be effective for symptom management in the setting of significant $\mathrm{RV}$ volume loading. ARBs have not proven beneficial in improving ventricular function, 


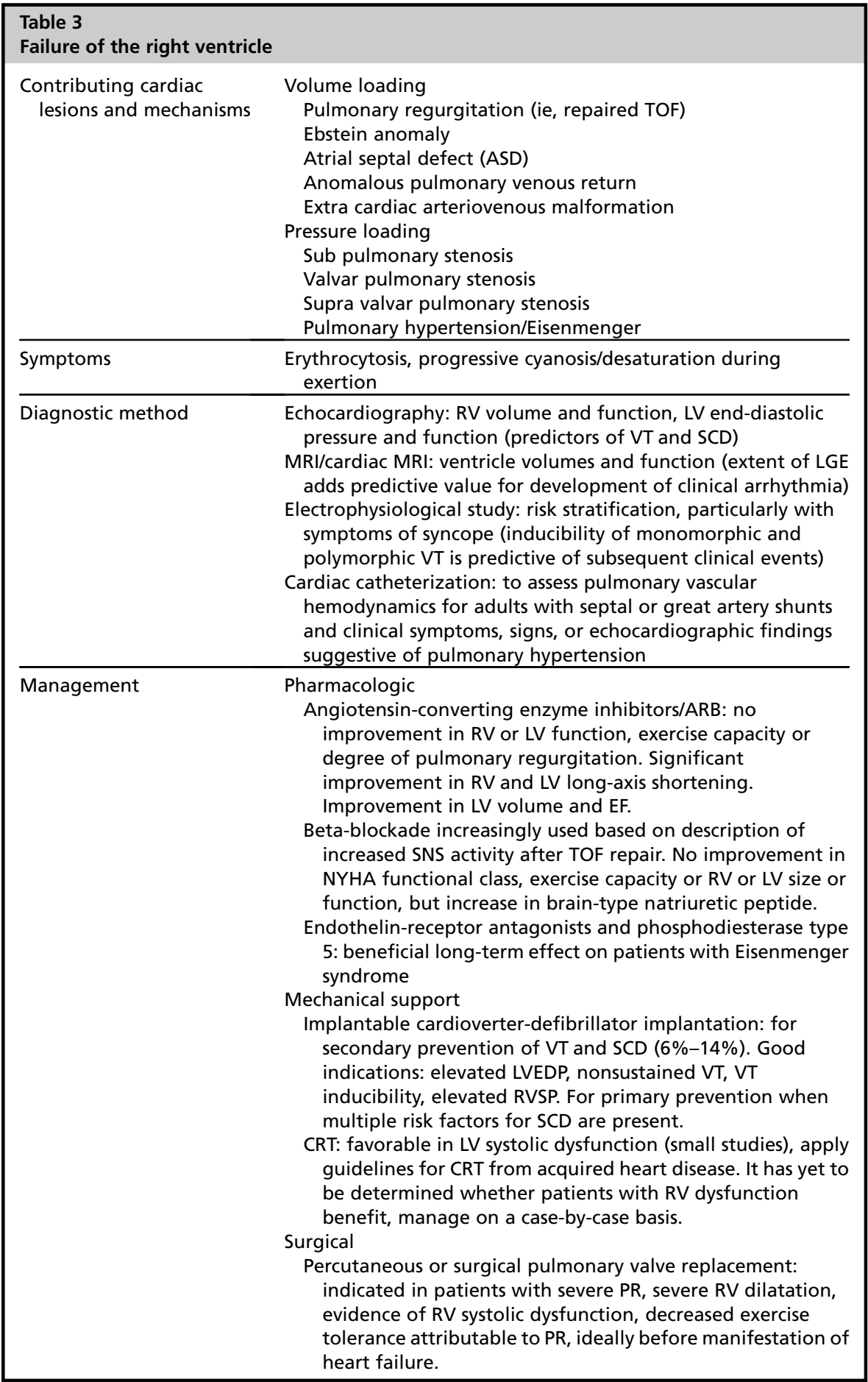

Abbreviations: CRT, cardiac resynchronization therapy; TOF, tetralogy of Fallot. 
exercise capacity, NT-proBNP levels, or clinical symptoms of right-sided heart failure. Similarly, there is a lack of evidence showing any benefits with the use of angiotensinconverting enzyme inhibitors and beta-blockers in right-sided heart failure. ${ }^{3,5,14,15,17}$

\section{HEART FAILURE IN SPECIFIC LESIONS Heart Failure in Patients with Fontan Procedure}

There are a number of anatomic variations resulting in a single ventricle physiology (Table 4). In the newborn, the primary goal of surgical therapy is to establish sufficient

\begin{tabular}{|c|c|}
\hline $\begin{array}{l}\text { Contributing cardiac } \\
\quad \text { lesions and mechanisms }\end{array}$ & $\begin{array}{l}\text { Fontan failure with reduced ejection fraction } \\
\text { Fontan failure with preserved ejection fraction }\end{array}$ \\
\hline Symptoms & $\begin{array}{l}\text { Protein-losing enteropathy, plastic bronchitis, portal } \\
\text { hypertension, peripheral edema, thrombus/pulmonary } \\
\text { embolism/stroke, cyanosis, atrial and ventricular arrhythmia }\end{array}$ \\
\hline Diagnostic method & $\begin{array}{l}\text { Regular clinical evaluation: heart failure should be anticipated } \\
\text { in the long-term care } \\
\text { Important to assess potentially reversible causes of heart failure } \\
\text { such as ventricular dysfunction, arrhythmias, thrombus in the } \\
\text { Fontan pathways, protein-losing enteropathy, valvular } \\
\text { dysfunction, residual right-to-left shunt, inflow or outflow } \\
\text { obstruction including a restrictive atrial or ventricular septal } \\
\text { defect that can impede cardiac output, elevated systemic } \\
\text { vascular resistance, elevated systemic venous pressures and } \\
\text { pulmonary vascular resistance, plastic bronchitis } \\
\text { Electrocardiogram: monitoring for bradycardia or sinus node } \\
\text { dysfunction } \\
\text { Stress testing: for assessing symptoms suggestive of } \\
\text { myocardial ischemia and heart failure } \\
\text { MRI or computed tomography scan: evaluation of common } \\
\text { long-term complications of atrial or arterial switch } \\
\text { Cardiac catheterization: invasive anatomic and hemodynamic } \\
\text { assessment, coronary patency }\end{array}$ \\
\hline Management & $\begin{array}{l}\text { Pharmacologic } \\
\text { Angiotensin-converting enzyme inhibitors: no significant } \\
\text { differences in SVR, resting Cl, diastolic function, exercise } \\
\text { capacity, ventricular size, Ross heart failure class, brain-type } \\
\text { natriuretic peptide levels, ejection fraction or mortality } \\
\text { (evidence: few studies, nonadult) } \\
\text { Beta-blockade: negative or neutral effect on symptoms or } \\
\text { clinical parameters } \\
\text { Diuretics and digoxin: widely used (evidence: not empirical) } \\
\text { Pulmonary vasodilator therapy: mixed results (improved peak } \\
V_{\mathrm{O}_{2}} \text { during exercise, increased pulmonary and systemic } \\
\text { blood flow at peak exercise, doppler derived myocardial } \\
\text { performance index, systolic arterial and ventricular } \\
\text { elastance, ventilatory efficiency (evidence: small studies) } \\
\text { Endothelin antagonists: unknown (evidence: studies ongoing) } \\
\text { Mechanical support } \\
\text { CRT: maintenance of atrioventricular synchrony (evidence: } \\
\text { case series) } \\
\text { Surgical }\end{array}$ \\
\hline
\end{tabular}

Abbreviations: CRT, cardiac resynchronization therapy; SVR, systemic vascular resistance. 
pulmonary blood flow to reach an acceptable level of oxygenation. This target is usually reached via surgical creation of a shunt to improve pulmonary blood flow. Thereafter, a surgical connection between the superior and inferior vena cava and the pulmonary artery is created. This so-called Fontan circulation is based on a passive flow of blood through the pulmonary circulation, strictly depending on the pressure gradient between central venous and left atrial pressure.

Over time, up to $40 \%$ of patients with a Fontan circulation develop heart failure. ${ }^{21}$ The underlying mechanisms are variable. Fontan failure can occur with depressed ejection fraction or with normal ejection fraction (primary diastolic failure). Diastolic failure of the single ventricle is commonly accompanied by valve insufficiency, and arrhythmias are regularly observed. Hebson and colleagues ${ }^{22}$ showed that patients with circulatory failure regularly develop elevated central venous and pulmonary capillary wedge pressures with low systemic vascular resistance and a preserved cardiac index. This hemodynamic profile is comparable with that of patients suffering from portal hypertension. However, in a Fontan circulation the ability to augment cardiac output above a certain threshold is limited. The increased postsinusoidal pressure in the inferior vena cava leads to liver fibrosis and to a portal hypertensive type circulatory derangement, resulting in vasoconstriction of the renal vasculature, sodium/water retention, edema and ascites. Patients with a systemic right ventricle face both limitations of a systemic right ventricle and failing Fontan pathophysiology.

If a failing Fontan circulation is present, a complete cardiac workup is recommended to identify correctable hemodynamic disturbance. ${ }^{3,22}$ However, pharmacologic heart failure treatment is rather difficult. The maintenance of ventricular contractility, treatment of arrhythmias and optimized balance between preload and afterload remain the most relevant targets.

The Fontan circulation depends on low pulmonary vascular resistance. A sustained increase in pulmonary vascular resistance, and thus afterload, should be treated. Studies with phosphodiesterase type 5 inhibitors such as sildenafil have shown some benefit (increase in peak pulmonary blood flow, peak $\mathrm{Vo}_{2}$, cardiac index, and stroke volume during exercise). Similar results have emerged in studies using bosentan (an endothelin-receptor antagonist). More recently, studies involving newer generation pulmonary vasodilators in Fontan patients have been initiated. . $^{3,13-15,17}$

To preserve ventricular contractility and an adequate preload, aggressive treatment of any arrhythmia is recommended. In the event of dyssynchronous contraction of the functional systemic ventricle, pacemaker therapy should be considered. Diuretics are effective for inappropriate fluid retention, such as edema, pleural effusion, and ascites. After initiation of beta-blockade in children and adolescents with a failing Fontan circulation, decreased requirements for diuretics were demonstrated in addition to increased ventricular ejection fraction. ${ }^{3,14}$ The effect of beta-blockers on the systemic vascular resistance has shown to be neutral in large studies. ${ }^{22}$ Neurohormonal blockade in Fontan failure has not shown benefit. The use of angiotensin-converting enzyme/ARB was not associated with improvements in cardiac index, exercise capacity, or diastolic filling patterns. ${ }^{23}$

\section{Heart Failure in Patients after Surgery for Transposition of the Great Arteries}

In patients with congenital TGA, the arterial switch operation is nowadays the standard of care. After the arterial switch, the LV acts as the systemic ventricle (anatomic correction). However, if a switch operation is not possible, the Senning or Mustard procedure (atrial switch) is performed.

Recently, Couperus and colleagues ${ }^{24}$ described the long-term follow-up of up to more than 30 years after atrial correction in 76 adult patients with TGA. Survival was 
$82 \%$ at 39.7 years postoperatively and exceeded 50 years in 4 patients. The most common complication was supraventricular tachycardia in more than $50 \%$ of patients. Heart failure was observed in $19 \%$ of patients 30 years after surgery. RV function was depressed in 31 (46\%) patients, and NYHA class was II or greater in 34 patients (48\%). Bradyarrhythmia, supraventricular tachycardia, and ventricular arrhythmia were associated with depressed RV and functional capacity. Ventricular arrhythmias, heart failure and surgical reinterventions were common during late follow-up. Heart failure therapy in these patients is therefore primarily based on antiarrhythmic therapy and preservation of RV function.

\section{PERIOPERATIVE CARE FOR ADULT PATIENTS WITH CONGENITAL HEART DISEASE- RELATED HEART FAILURE}

The number of adults with CHD now surpasses the number of children with $\mathrm{CHD},{ }^{25}$ leading to the fact that adults with complex CHD managed for noncardiac surgery are not restricted to highly specialized centers. Agarwal and colleagues ${ }^{9}$ reported that $27.1 \%$ of ACHD admissions to small-sized hospitals represented complex ACHD. In the United States, general anesthesia knowledge and training standards for ACHD are lacking, resulting in a low level of knowledge and comfort with providing perioperative and obstetric care for patients with ACHD, even in academic centers. ${ }^{26}$

Cardiac and noncardiac surgical procedures in patients with CHD carry an increased risk for perioperative adverse events. Factors judged to have a major contribution to adverse events were the nature of $\mathrm{CHD}(50 \%)$, preoperative assessment or optimization (40\%), intraoperative anesthetic care $(55 \%)$, and postoperative monitoring/care $(50 \%){ }^{27}$

\section{Preoperative Risk Assessment}

The risk of patients with ACHD generally depends on 4 factors: the underlying nature of $\mathrm{CHD}$, the individual course of this CHD, associated noncardiac morbidity, and the type of the noncardiac surgical procedure. Because of the complexity of morbidity and the individual course of disease, individualized preoperative risk assessment is necessary. This process includes assessment, optimization, and care by a multidisciplinary team with a detailed understanding of the patient's cardiac defect, functional status, and anticipation of the perioperative stresses ${ }^{5,28}$ (Box 2).

\section{Heart Failure}

Clinical performance may be misleading as a relevant part of the ACHD may present with good physical performance. Proven predictive tools for evaluation of the ventricular function are echocardiography and the use of biomarkers such as NT-proBNP. An NT-proBNP of greater than $33.3 \mathrm{mmol} / \mathrm{L}$ is strongly associated with cardiac events, death, or heart failure, independent of echocardiography findings. ${ }^{29}$ The risk of patients with a high level of NT-proBNP could be further differentiated by adding hs-TnT and growth differentiation factor 15. Patients with elevated levels of all 3 biomarkers are at highest risk of cardiac events, death, and heart failure. ${ }^{29}$ Low biomarker levels were accurately associated with a low risk of heart failure and death. ${ }^{29}$ These findings could enable accurate preoperative identification of patients at high perioperative cardiac risk and allow for the timely initiation or expansion of risk-reducing strategies.

\section{Arrhythmias}

Almost $50 \%$ of all ACHD will develop atrial tachyarrhythmias during their lifetime. ${ }^{30}$ Factors such as underlying anatomy, age, and surgical repair technique have an 


\section{Box 2}

\section{Recommendations for preoperative risk assessment in ACHD}

1. Basic preoperative assessment for patients with ACHD should include systemic arterial oximetry, an electrocardiogram, chest radiographs, transthoracic echocardiography, and blood tests for full blood count and coagulation screen. (LOE: C)

2. It is recommended that when possible, the preoperative evaluation and surgery for patients with ACHD be performed in a regional center specializing in congenital cardiology, with experienced surgeons and cardiac anesthesiologists. (LOE: C)

3. Certain high-risk patient populations should be managed at centers for the care of patients with ACHD under all circumstances, unless the operative intervention is an absolute emergency. High-risk categories include patients with the following:
a. Prior Fontan procedure.
b. Severe pulmonary arterial hypertension.
c. Cyanotic CHD.
d. Complex CHD with residua such as heart failure, valve disease, or the need for anticoagulation.
e. Patients with CHD and malignant arrhythmias. (LOE: C)

4. Consultation with ACHD experts regarding the assessment of risk is recommended for patients with CHD who will undergo noncardiac surgery. (LOE: C)

5. Consultation with a cardiac anesthesiologist is recommended for moderate- and high-risk patients. (LOE: C)

Abbreviation: LOE, level of evidence.

Adapted from Warnes CA, Williams RG, Bashore TM, et al. ACC/AHA 2008 guidelines for the management of adults with congenital heart disease: a report of the American College of Cardiology/American Heart Association Task Force on Practice Guidelines (Writing Committee to Develop Guidelines on the Management of Adults With Congenital Heart Disease). Developed in Collaboration With the American Society of Echocardiography, Heart Rhythm Society, International Society for Adult Congenital Heart Disease, Society for Cardiovascular Angiography and Interventions, and Society of Thoracic Surgeons. J Am Coll Cardiol 2008;52(23):e143e263; with permission.

impact on prevalence and arrhythmia substrate. Atrial tachyarrhythmia, mostly in the form of intra-atrial reentrant tachycardia and atrial fibrillation, are more frequent than ventricular tachyarrhythmias. The presence of atrial tachyarrhythmia significantly increases the risk of heart failure and stroke. ${ }^{31}$ For perioperative management, common scores for thromboembolic risk calculation, like CHADS2 and CHA2DS2-VASc, are not predictive for thromboembolic risk in patients with ACHD. ${ }^{30,31}$ The complexity of $\mathrm{CHD}$, however, was independently associated with thromboembolic events. ${ }^{32}$ Antiarrhythmic therapy with class IC antiarrhythmic agents and amiodarone is effective in almost $50 \%$ of the patients, although systemic side effects and proarrhythmic properties being of concern. ${ }^{30,32}$ Electrical cardioversion with appropriate anticoagulation is safe and effective. ${ }^{30,32}$ Catheter ablation procedures and the MAZE procedure for drug-refractory atrial fibrillation may be beneficial in selected patients, but experience is limited. ${ }^{32}$ Antiarrhythmic therapy is effective in suppressing ventricular arrhythmias, but has not been associated with improved survival. ${ }^{28}$

\section{PREGNANCY}

The overall incidence of maternal CHD is $0.6 \%{ }^{33}$ and accounts for $60 \%$ to $80 \%$ of abnormalities in pregnant women with structural heart disease. ${ }^{34}$ The incidence of cardiac complications in pregnancies associated with CHD is estimated to be around 
$11 \%$, but may be as high as $20 \% .^{33,35}$ Heart failure is the most common cardiac complication during pregnancy with 2 peaks of onset. The first peak is between 23 and 30 weeks, when most of the hemodynamic changes have taken place and the second peak around delivery. If heart failure affects a woman with CHD during pregnancy, maternal mortality today is around $4.8 \% .^{33-35}$

Women with CHD should receive pregnancy counseling with input from an ACHD cardiologist to determine maternal cardiac, obstetric and fetal risks, and long-term risks to the mother. Then an individualized plan of care should be developed. During pregnancy, women with CHD (especially those with complex forms of ACHD) should be managed collaboratively by cardiologists, obstetricians, and anesthesiologists who are all experienced in ACHD. ${ }^{5}$

To assess the maternal cardiovascular risk, the Canadian Cardiac Disease in Pregnancy and Zwangerschap bij Aangeboren HARtAfwijkingen (ZAHARA) scores are used, along with the modified World Health Organization classification of maternal cardiovascular risk. ${ }^{36}$ Pregnancy is generally contraindicated in women at high risk of maternal morbidity and mortality, which includes women with pulmonary arterial hypertension, severe left-sided obstructive lesions, significant systemic ventricular dysfunction (ejection fraction of $<30 \%$ ) and NYHA functional class III or IV.

Medical management involves optimizing heart failure medications, in particular the discontinuation of agents toxic to the fetus while titrating nontoxic heart failure medications. ${ }^{37}$ If well-managed, vaginal delivery with adequate analgesia is well-tolerated in most obstetric patients with CHD. ${ }^{37}$

For intrapartum monitoring, telemetry, oxygen saturation monitoring, and invasive hemodynamic monitoring should be considered on an individualized basis. ${ }^{36,37}$ Postpartum monitoring of the patient with CHD with heart failure or at high risk for heart failure requires telemetry monitoring for at least 24 hours postpartum, as well as careful clinical monitoring for evidence of decompensated heart failure and development of arrhythmia. The risk for recurrent or worsening heart failure can occur up to 8 weeks after delivery. 37

\section{NONMEDICAL THERAPY FOR REFRACTORY HEART FAILURE}

In refractory and end-stage heart failure, mechanical circulatory support remains one of the final treatment options. There is a wide spectrum of techniques ranging from acute implementation of temporary venovenous or venoarterial extracorporeal membrane oxygenation to continuous treatment with implantable mechanical assist devices as a bridge to recovery or even as a bridge to heart transplantation. It is estimated that $10 \%$ to $20 \%$ of patients with ACHD will require heart transplantation at some point in their life. ${ }^{38}$

In 2018, Esteve-Ruiz and co-workers presented the results of a series of 10 patients with ACHD undergoing heart transplantation. All of these patients suffered from failing Fontan circulation or another form of single ventricle pathology. Nine of the 10 patients were transplanted electively, whereas 1 patient required extracorporeal membrane oxygenation preoperatively. Compared with patients with heart failure from non-CHD, both short-term mortality and postoperative stay in the intensive care unit were increased. After 1 year, outcomes were comparable between the 2 groups. ${ }^{39}$ However, these series of individual patient cases is too small to draw definitive conclusions on whether or not heart transplantation is suitable in patients with ACHD. In an analysis by Goldberg and colleagues, patients with $\mathrm{ACHD}$ represented $2.5 \%$ of all patients placed on the US heart transplantation list. ${ }^{40}$ 
Although there is no evidence-based consensus on the role of heart transplantation in patients with ACHD, the use of ventricular assist devices in these patients is an established standard of care. In experienced centers, the mortality of ventricular assist device implantation in patients with ACHD is comparable to mortality in non-patients with ACHD, although this only holds true for patients with a 2-ventricle anatomy. There are no systematic studies addressing the use of mechanical support systems in patients with single ventricle physiology (Fontan circulation), although some information is available from case series or individual case reports.

\section{SUMMARY}

Owing to advances in care, almost $90 \%$ of children born with a CHD grow up into adulthood and many achieve elderly age. Although many cardiac birth defects can be surgically well-managed, residual anomalies eventually give rise to complications and increase the risk of severe morbidity and mortality. Because of their heterogeneity and the complexity of the pathophysiologic profile, care for these patients is very challenging. With the increasing number of adults with $\mathrm{CHD}$ and the increasing complexity with aging of these patients, there is a growing need for multidisciplinary care programs and guidelines. However, most guidelines are based on studies with patients without CHD and should be extrapolated with care when used for patients with CHD, taking into account the individual pathophysiology. In the follow-up of adults with CHD and in the perioperative care of these patients, special attention should be paid to signs and symptoms of developing heart failure and to prevention of deteriorating heart function and arrhythmias for these are major causes of adverse cardiac events and mortality. Also, pregnant women with ACHD who may clinically seem to have normal heart function are at increased risk, mainly owing to hematologic and cardiovascular changes related to pregnancy. Challenges for the near future are to improve knowledge on optimal diagnostics and pharmacologic treatment, adequate timing of interventions, and education of health care teams.

\section{REFERENCES}

1. Mitchell SC, Korones SB, Berendes HW. Congenital heart disease in 56,109 births incidence and natural history. Circulation 1971;43(3):323-32.

2. van der Bom T, Bouma BJ, Meijboom FJ, et al. The prevalence of adult congenital heart disease, results from a systematic review and evidence based calculation. Am Heart J 2012;164(4):568-75.

3. Opina AD, Franklin WJ. Management of heart failure in adult congenital heart disease. Prog Cardiovasc Dis 2018;61(3-4):308-13.

4. van der Linde D, Konings EEM, Slager MA, et al. Birth prevalence of congenital heart disease worldwide. J Am Coll Cardiol 2011;58(21):2241-7.

5. Stout KK, Daniels CJ, Aboulhosn JA, et al. 2018 AHA/ACC guideline for the management of adults with congenital heart disease. A report of the American College of Cardiology/American Heart Association Task Force on Clinical Practice Guidelines. Circulation 2019;139(14):e698-800.

6. Hoffman JIE, Kaplan S, Liberthson RR. Prevalence of congenital heart disease. Am Heart J 2004;147(3):425-39.

7. Moussa NB, Karsenty C, Pontnau F, et al. Characteristics and outcomes of heart failure-related hospitalization in adults with congenital heart disease. Arch Cardiovasc Dis 2017;110(5):283-91.

8. Afilalo J, Therrien J, Pilote L, et al. Geriatric congenital heart disease. J Am Coll Cardiol 2011;58(14):1509-15. 
9. Agarwal S, Sud K, Menon V. Nationwide hospitalization trends in adult congenital heart disease across 2003-2012. J Am Heart Assoc 2016;5(1) [pii:e002330].

10. Zomer A, Vaartjes I, van der Velde E. Heart failure admissions in adults with congenital heart disease; risk factors and prognosis. Int J Cardiol 2013;168: 2487-93.

11. Verheugt CL, Uiterwaal CSPM, van der Velde ET, et al. Mortality in adult congenital heart disease. Eur Heart J 2010;31(10):1220-9.

12. Bolger AP, Gatzoulis MA. Towards defining heart failure in adults with congenital heart disease. Int J Cardiol 2004;97:15-23.

13. Krieger EV, Valente AM. Heart failure treatment in adults with congenital heart disease: where do we stand in 2014? Heart 2014;100(17):1329-34.

14. Stout KK, Broberg CS, Book WM, et al. Chronic heart failure in congenital heart disease: a scientific statement from the American Heart Association. Circulation 2016;133(8):770-801.

15. Warnes CA, Williams RG, Bashore TM, et al. ACC/AHA 2008 guidelines for the management of adults with congenital heart disease. J Am Coll Cardiol 2008; 52(23): e143-263.

16. Ponikowski P, Voors AA, Anker SD, et al. 2016 ESC guidelines for the diagnosis and treatment of acute and chronic heart failure: the task force for the diagnosis and treatment of acute and chronic heart failure of the European Society of Cardiology (ESC) Developed with the special contribution of the Heart Failure Association (HFA) of the ESC. Eur Heart J 2016;37(27):2129-200.

17. Yancy CW, Jessup M, Bozkurt B, et al. 2017 ACC/AHA/HFSA focused update of the 2013 ACCF/AHA guideline for the management of heart failure. J Card Fail 2017:23(8):628-51.

18. Bredy C, Ministeri M, Kempny A, et al. New York Heart Association (NYHA) classification in adults with congenital heart disease: relation to objective measures of exercise and outcome. Eur Heart J Qual Care Clin Outcomes 2018;4(1):51-8.

19. Rudski LG, Lai WW, Afilalo J, et al. Guidelines for the echocardiographic assessment of the right heart in adults: a report from the American Society of Echocardiography. J Am Soc Echocardiogr 2010;23(7):685-713.

20. Budts W, Roos-Hesselink J, Rädle-Hurst T, et al. Treatment of heart failure in adult congenital heart disease: a position paper of the Working Group of Grown-Up Congenital Heart Disease and the Heart Failure Association of the European Society of Cardiology. Eur Heart J 2016;37(18):1419-27.

21. Norozi K, Wessel A, Alpers V, et al. Incidence and risk distribution of heart failure in adolescents and adults with congenital heart disease after cardiac surgery. Am J Cardiol 2006;97:1238-43.

22. Hebson CL, McCabe NM, Elder RW, et al. Hemodynamic phenotype of the failing Fontan in an adult population. Am J Cardiol 2013;112(12):1943-7.

23. Ohuchi $\mathrm{H}$. Adult patients with Fontan circulation: what we know and how to manage adults with Fontan circulation? J Cardiol 2016;68:181-9.

24. Couperus LE, Vliegen HW, Zandstra TE, et al. Long-term outcome after atrial correction for transposition of the great arteries. Heart 2019;105(10):790-6.

25. Gilboa SM, Devine OJ, Kucik JE, et al. Congenital heart defects in the United States: estimating the magnitude of the affected population in 2010. Circulation 2016;134(2):101-9.

26. Maxwell BG, Williams GD, Ramamoorthy C. Knowledge and attitudes of anesthesia providers about noncardiac surgery in adults with congenital heart disease: survey of anesthesiologists caring for adults. Congenit Heart Dis 2014; $9(1): 45-53$. 
27. Maxwell BG, Posner KL, Wong JK, et al. Factors contributing to adverse perioperative events in adults with congenital heart disease: a structured analysis of cases from the closed claims project: adverse perioperative events in ACHD. Congenit Heart Dis 2015;10(1):21-9.

28. Lovell AT. Anaesthetic implications of grown-up congenital heart disease. $\mathrm{Br} J$ Anaesth 2004;93(1):129-39.

29. Baggen VJM, van den Bosch AE, Eindhoven JA, et al. Prognostic value of N-Terminal Pro-B-Type natriuretic peptide, Troponin-T, and growth-differentiation factor 15 in adult congenital heart disease. Circulation 2017;135(3):264-79.

30. Bouchardy J, Therrien J, Pilote L, et al. Atrial arrhythmias in adults with congenital heart disease. Circulation 2009;120(17):1679-86.

31. Baehner T, Ellerkmann RK. Anesthesia in adults with congenital heart disease. Curr Opin Anaesthesiol 2017;30(3):418-25.

32. Hernández-Madrid A, Paul T, Abrams D, et al. Arrhythmias in congenital heart disease: a position paper of the European Heart Rhythm Association (EHRA), Association for European Paediatric and Congenital Cardiology (AEPC), and the European Society of Cardiology (ESC) Working Group on Grown-up Congenital heart disease, endorsed by HRS, PACES, APHRS, and SOLAECE. Europace 2018;20(11):1719-53.

33. Warrick CM, Hart JE, Lynch AM, et al. Prevalence and descriptive analysis of congenital heart disease in parturients: obstetric, neonatal, and anesthetic outcomes. J Clin Anesth 2015;27(6):492-8.

34. Westhoff-Bleck M, Hilfiker-Kleiner D, Pankuweit S, et al. Cardiomyopathies and congenital heart disease in pregnancy. Geburtshilfe Frauenheilkd 2018;78(12): 1256-61.

35. van Hagen IM, Boersma E, Johnson MR, et al. Global cardiac risk assessment in the Registry Of Pregnancy And Cardiac disease: results of a registry from the European Society of Cardiology: Cardiac risk assessment in the ROPAC. Eur J Heart Fail 2016;18(5):523-33.

36. Canobbio MM, Warnes CA, Aboulhosn J, et al. Management of pregnancy in patients with complex congenital heart disease: a scientific statement for healthcare professionals from the American Heart Association. Circulation 2017;135(8): e50-87.

37. Bradley EA, Saraf A, Book W. Heart failure in women with congenital heart disease. Heart Fail Clin 2019;15(1):87-96.

38. Schweiger M, Lorts A, Conway J. Mechanical circulatory support challenges in pediatric and (adult) congenital heart disease. Curr Opin Organ Transplant 2018;23(3):301-7.

39. Esteve-Ruiz I, Grande-Trillo A, Rangel-Sousa D, et al. Complex congenital heart disease: is heart transplantation an option? Transplant Proc 2018;50(2):655-7.

40. Goldberg SW, Fisher SA, Wehman B, et al. Adults with congenital heart disease and heart transplantation: optimising outcomes. J Heart Lung Transplant 2014; 33(9):873-7. 